\title{
Fabricating a Highly Sensitive QCM Sensor Using AAO Nanoholes and Its Application for Biosensing ${ }^{\dagger}$
}

\author{
Naoto Asai, Tomohiro Shimizu, Shoso Shingubara and Takeshi Ito * \\ Graduate School of Engineering Science, Kansai University, Osaka 564-8680, Japan; \\ * Correspondence: t.ito@kansai-u.ac.jp; Tel.: +81-06-6368-1121 \\ + Presented at the Eurosensors 2017 Conference, Paris, France, 3-6 September 2017. \\ Published: 9 August 2017
}

\begin{abstract}
A nanostructure composed of Anodic Aluminum Oxide (AAO) was obtained on an electrode of a quartz crystal microbalance (QCM) chip by anodizing $\mathrm{Al}$ thin film in an oxalic acid solution. The effective surface area was expanded by these nanostructures. Several morphologies were observed under various anodic conditions by using scanning electron microscopy (SEM). We demonstrated that a QCM chip with the AAO was effective in biosensing because of its large surface area. The frequency shift corresponding to an antigen-antibody reaction improved on the nanostructured electrode compared with a flat surface electrode.
\end{abstract}

Keywords: biosensing; QCM; anodic aluminum oxide; nanostructure

\section{Introduction}

In the recent years, many biosensing systems, for examples, surface plasmon resonance (SPR) and enzyme-linked immune sorbent assay (ELISA) are widely used. Both technics can detect analtyte with high sensitivity. However, they have disadvantages. In the SPR, it's low throughput technic and it needs complex optical equipment [1]. In the ELISA, it's time consuming method. Additionally, it needs professional skill and labeling material [2]. Therefore, some conditions are required for biosensing as follows; low cost equipment, rapid analysis, real time measurement, high sensitivity and convenient protocol. If these requires are achieved, we can perform biosensing system easily. In this study, we focused on a quartz crystal microbalance (QCM) method by following reasons. QCM method is one of promising approaches to detect gas molecules and bio materials. In addition, it has advantages in real time measurement, high sensitivity, and portability. However, the method has poor sensitivity to low molecular weight materials $(<1 \mathrm{kDa})$ because of its measuring principle, mass change. Theoretical mass change by absorption of analyte on the electrode over the quartz is estimated by using Sauerbrey equation [3]. In fact, the magnitude of the frequency shift is proportional to the mass change. For an example, the mass change is calculated as $1.07 \mathrm{ng}$ with the frequency shift of $1 \mathrm{~Hz}$ on the fundamental frequency of $9 \mathrm{MHz}$. On the analysis of $1 \mathrm{kDa}$ material, the frequency shift of $1 \mathrm{~Hz}$ is about $10^{-12} \mathrm{~mol}$. Its concentration is too low to detect. Some groups introduced to fabricate the nanostructures on the electrode of QCM chip to improve the sensitivity for the detection of the low molecular weight materials. For example, a nanostructured $\mathrm{IrO}_{2}$ was manufactured on the gold electrode by metal organic chemical vaper deposition (MOCVD) [4]. The sensor could detect volatile organic compounds (VOC). However, it was difficult to control the nanostructures of $\mathrm{IrO}_{2}$, and the material was hazardous. The nanostructured QCM dynamically monitored antimicrobial resistance using magnesium zinc oxide [5]. As the results, the surface area was enlarged by nanostructures, and the amount of analyte binding on the surface was greatly improved. However, the experimental protocol wasn't convenience because the protocol labeled the bacteria to antimicrobial on the electrode. Here, we focused on nano porous structure of anodic aluminum oxide (AAO) to detect the analyte with highly sensitivity. AAO is obtained by only 
anodizing under a suitable potential in electrolytic solution [6]. Then, self-organized nanoholes having a triangle lattice is formed in a downward direction with high aspect ratio. And the aspect ratio is controllable by anodic condition, for example type of electrolyte solution, applying potential, anodization time and solution temperature [7]. In this study, we attempted to fabricate AAO nanostructure on the electrode of quartz crystal, and the surface area was estimated. The sensitivity on the antigen-antibody interaction was evaluated by using Anti-Mouse IgG and Mouse IgG in the QCM measurement.

\section{Materials and Methods}

All the reagents used here were purchased from Woko Pure Chemicals Co. Ltd (Osaka, Japan). Firstly, titanium $(\mathrm{Ti}, 20 \mathrm{~nm})$ and aluminum $(\mathrm{Al}, 500 \mathrm{~nm})$ was sputtered on a quartz crystal in this order which fundamental frequency was $9 \mathrm{MHz}$. Secondly, Al thin film was anodized under the applied voltage of $40 \mathrm{~V}$ in $0.3 \mathrm{M}$ oxalic acid for 1 to $4 \mathrm{~min}$. Finally, a diameter of nanohole was widen in $5 \mathrm{wt} \%$ phosphoric acid. Then, we obtained QCM chip with AAO nanostructure on the surface of the quartz. The morphology of AAO nanostructure was observed by scanning electron microscopy (SEM; JEOL JSM-7500F). A preparation for measuring antigen-antibody interaction was explained as follows:

Self-assembled monolayer (SAM) consist of APTES (3-aminopropyltriethoxysilane) was formed on the surface of the AAO nanostructure. Anti-Mouse IgG $(100 \mu \mathrm{g} / \mathrm{mL})$ and Bovine serum albumin (BSA; $0.01 \%$ ) were immobilized on the surface of AAO nanostructure through SAM for $10 \mathrm{~min}$, in this order. Then, the QCM chip was set on a measurement cell. Finally, Mouse IgG $(10 \mu \mathrm{g} / \mathrm{mL})$ was injected in the measurement cell and monitored a frequency shift of the QCM.

\section{Result and Discussion}

Figure 1a, $\mathrm{b}$ show cross and surface sectional SEM images of AAO nanostructure on the quartz crystal. The AAO was fabricated under the voltage of $40 \mathrm{~V}$ for $4 \mathrm{~min}$. At first, the aluminum thin film was sputtered $500 \mathrm{~nm}$. While, it decreased to be about $100 \mathrm{~nm}$, and a thickness of the obtained AAO nanostructure was $450 \mathrm{~nm}$ after anodization. The diameter of nanohole was increased to be $50-60 \mathrm{~nm}$ by etching the AAO using phosphoric acid. The estimated hole density was 100 piece per square $\mu \mathrm{m}$.

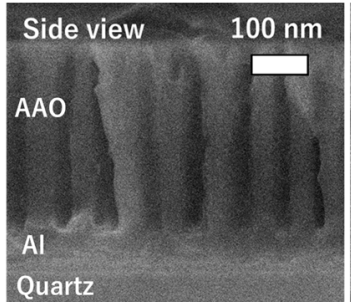

(a)

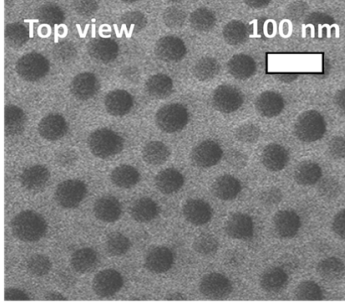

(b)

Figure 1. Cross sectional (a) and surface (b) SEM images of AAO nanostructure on the quartz crystal. Applied voltage was set as $40 \mathrm{~V}$ for $4 \mathrm{~min}$ in the oxalic acid solution.

Table 1 shows the ratio of surface area compared with non-anodic electrode, flat surface. The values were calculated using the Equation (1) as follows. The surface area of the nanostructure was estimated using the length, radius and density of the nanohole, and was ranged from 3.1 to 9.3.

$$
\text { Ratio of surface area }=\frac{\text { Surface of AAO naostructure }}{\text { non }- \text { anodic electrode }}
$$

Figure 2 shows conductance measurement depending on the preparation process of QCM measurement. The frequency shift decreased for each process in order. The results indicated the QCM chip was affected by each mass changes. Therefore, Anti-Mouse IgG and BSA were immobilized on the surface of AAO nanostructure normally through amino groups on the SAM. Figure 3 shows a frequency shift of the QCM measurement in the antigen-antibody interaction depending on the ratio of the surface area. The obtained frequency shifts were approximately $50,100,200,500 \mathrm{~Hz}$ on each 
AAO nanostructured QCM chip. The magnitude of frequency shift increased with the ratio of the surface area. The result indicates that sensitivity of the AAO nanostructured QCM chip was improved by the nanostructure and the obtained frequency shift was 10 times larger than that on the flat surface.

Table 1. Estimated ratio of surface area.

\begin{tabular}{cccccc}
\hline Anodic time (min) & 0 & 1 & 2 & 3 & 4 \\
\hline Ratio of surface area & 1.0 & 3.1 & 5.1 & 7.2 & 9.3 \\
\hline
\end{tabular}

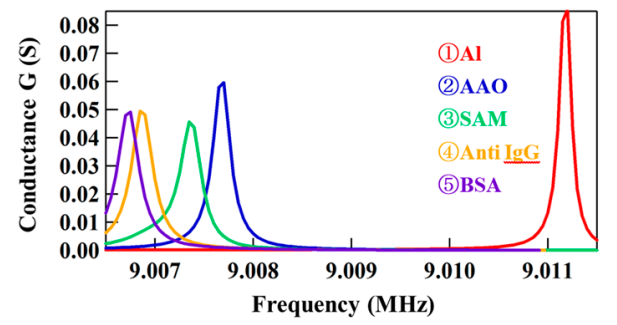

Figure 2. The conductance measurement of the AAO nanostructured QCM chip under applied voltage of $40 \mathrm{~V}$ for $4 \mathrm{~min}$ for each preparation process.

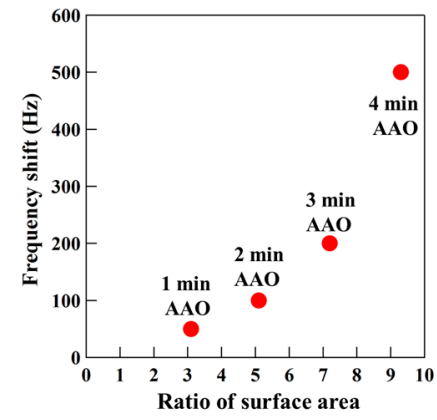

Figure 3. Frequency shift of the antigen-antibody interaction using the QCM with AAO nanostructure. Concentration of antigen (mouse $\operatorname{IgG}$ ) was $10 \mu \mathrm{g} / \mathrm{mL}$ in the all experiments.

\section{Conclusions}

An aluminum was sputtered on the electrode of QCM chip, and it was anodized in the oxalic acid solution. Then, it succeeded to fabricate AAO nanostructured QCM chip having high aspect nanoholes array. The diameter of nanohole was ranged from 50 to $60 \mathrm{~nm}$. The density of the nanoholes was approximately 100 pieces $/ \mu \mathrm{m}^{2}$. The surface area of AAO nanostructure by applied voltage of $40 \mathrm{~V}$ for $4 \mathrm{~min}$ was enlarged approximately 9.3 times comparing with non-anodic electrode QCM chip. In the QCM measurement with antigen-antibody interaction, the obtained frequency shift was $500 \mathrm{~Hz}$ as the maximum value. Comparing AAO nanostructured and non-anodic QCM chip, the sensitivity was improved by large surface area of AAO.

Acknowledgments: This research was supported by the Formation of research center at Kansai University, and was partially conducted by MEXT-Supported Program for the Strategic Research Foundation at Private Universities, "Creation of 3D nano-micro structures and its application to biomimetics and medicine", 2015-2019.

Conflicts of Interest: The authors declare no conflict of interest.

\section{References}

1. Liedberg, B.; Nylander, C.; Lundstrom, I. Surface plasmon resonance for gas detection and biosensing. Sens. Actuator 1983, 4, 299-304, doi:10.1016/0250-6874(83)85036-7.

2. Engvall, E.; Perlmann, P. Enzyme-linked immunosorbent assay (ELISA) quantitative assay of immunoglobulin G. Immunochemistry 1971, 8, 871-874, doi:10.1016/0019-2791(71)90454-X. 
3. Saurbrey, G. The use of quartz oscillators for weighing thin layers and for Microweighing. Z. Phys. 1955, 155, 296-222, NAID: 10029110884.

4. Chao, T.W.; Liu, C.J.; Hsieh, A.H.; Changb, H.M.; Huang, Y.S.; Tsai, D.S. Quartz crystal microbalance sensor based on nanostructured IrO2. Sens. Actuators B Chem. 2007, 122, 95-100, doi:10.1016/j.snb.2006.05.009.

5. Reyes, P.I.; Yang, K.; Zheng, A.; Li, R.; Li, G.; Lu, Y.; Tsang, C.K.; Zheng, S.X.F. Dynamic monitoring of antimicrobial resistance using magnesium zinc oxide nanostructure-modified quartz crystal microbalance. Biosens. Bioelectron. 2017, 93, 189-197, doi:10.1016/j.bios.2016.09.011.

6. Masuda, H.; Yada, K.; Osaka, A. Self-Ordering of Cell Configuration of Anodic Porous Alumina with Large-Size Pores in Phosphoric Acid Solution. Jpn. J. Appl. Phys. 1998, 37, 1340-1342, IOP: 1374065/37/11A/L1340.

7. Shimizu, T.; Xie, T.; Nishikawa, J.; Shingubara, S.; Senz, S.; Gosele, U. Synthesis of Vertical High-Density Epitaxial Si(100) Nanowire Arrays on a Si(100) Substrate Using an Anodic Aluminum Oxide Template. Adv. Mater. 2007, 19, 917-920, doi:10.1002/adma.200700153.

(C) 2017 by the authors. Licensee MDPI, Basel, Switzerland. This article is an open access article distributed under the terms and conditions of the Creative Commons Attribution (CC BY) license (http://creativecommons.org/licenses/by/4.0/). 